Method Retrospective extraction of data for all CT/GC TMA testing between 2011-2014, and analysis of selected records where TS \& urogenital \pm rectal sites sampled at the same visit. Results CT was detected on TS from 1.2\% of female (adding 8 extra cases), and from $0.76 \%$ of MSM (adding no extra cases) GC was detected on TS from $0.76 \%$ of females (adding 9 extra cases), and from $3.0 \%$ of males (adding 3 extra cases) In a subset of 251 females who had RS, GC was detected on TS from $3.6 \%$ adding 5 extra cases to the 4 urogenital/rectal diagnoses.

Conclusions In diagnosis of CT infection, TS find only a few extra male \& female cases For GC infection TS did increase the number of diagnoses (females from 12 to 21, males from 17 to 20). Whilst GC testing by TMA adds no cost to a genital sample which is already being tested for CT, a throat swab which is an extra sample has to be charged at full price by the Laboratory. Perhaps full price testing of TS can only be justified for groups with higher than average GC diagnoses such as MSM (3.0\%) or females with an indication for a rectal testing $(3.6 \%)$.

\section{P027 A QUALITATIVE STUDY EXPLORING THE POTENTIAL INFLUENCES OF SEXUALITY, GENDER IDENTITY, AND OCCUPATION ON HEALTH STATES AND ENGAGEMENT WITH HEALTHCARE AMONG LGBTQIA+ SEX WORKERS IN NEW ZEALAND}

Emma Dorothy Mills*, Chris Burton. University of Aberdeen, Aberdeen, UK

\subsection{6/sextrans-2016-052718.81}

Background/introduction The stigma an individual experiences in relation to their occupation, gender identity, or sexuality adversely influences health and well-being. There is however, limited literature which explores the joint influence of both sex work (a highly stigmatised occupation), and a sexual minority identity on health states and engagement with services. Preliminary work suggested that such individuals face greater risk of illhealth, and of experiencing barriers to care.

Aim(s)/objectives To explore how LGBTQIA $^{+}$sex workers perceive their occupation, sexuality, and gender identities influence their health states and access to health care.
Methods Semi-structured phone interviews were conducted with sex workers who self-identified as LGBTQIA ${ }^{+}$. Purposive sampling of participants ensured individuals were diverse in their sexuality, gender identity, and type and duration of sex work experience. The data collected during these interviews was analysed using a thematic approach.

Results Seven interviews were conducted. It was apparent that continuing social stigma directed towards sex workers and members of the LGBTQIA $^{+}$community perpetuates occupational hazards and acts as a barrier to accessing healthcare. The positive influences of a community of stigmatised peers in promoting engagement with health services was explored, including community information sharing networks and providing specific services inclusive to the needs of LGBTQIA $^{+}$sex workers.

Discussion/conclusion Whilst decriminalisation has reduced the stigma faced by many sex workers in New Zealand, disproportionate discrimination persists among those who identify as LGBTQIA $^{+}$, negatively impacting health states. The utilisation of peer networks promoting access to healthcare within this community is requiring of further research.

\section{P028 DO WE REALLY NEED TO SEND AN MSU?}

Bethanie Rooke*, Alice Baker, Sarah Barrett. Whittall Street Clinic, Birmingham, UK

10.1136/sextrans-2016-052718.82

Background/introduction Midstream urine (MSU) results create a significant workload for our clinic. MSU can diagnose urinary tract infection (UTI), but detecting asymptomatic bacteriuria or contaminants confuses management. Lower UTI is common in non-pregnant women, but MSU is unnecessary as UTI can be diagnosed clinically. Local guidelines identified four indications for MSU: women with dysuria and loin pain, urinary symptoms in pregnancy, men with dysuria and frequency/urgency, and epididymo-orchitis.

$\operatorname{Aim}(\mathbf{s}) /$ objectives To assess whether MSU is requested for appropriate indications, and to evaluate the usefulness of MSU in diagnosing and managing patients in a sexual health clinic.

\begin{tabular}{|c|c|c|c|c|c|c|c|c|}
\hline \multirow[t]{2}{*}{$\begin{array}{l}\text { Urogenital/rectal } \\
\text { diagnosis }\end{array}$} & \multicolumn{4}{|c|}{$\begin{array}{l}\text { Females: } \\
\text { Throat swab results }\end{array}$} & \multicolumn{4}{|c|}{$\begin{array}{l}\text { Males: } \\
\text { Throat swab results }\end{array}$} \\
\hline & Neg & Pos & Total & $\%$ Positive & Neg & Pos & Total & \% Positive \\
\hline CT Negative & 2039 & 8 & 2047 & 0.39 & 240 & 0 & 240 & 0 \\
\hline CT Positive & 133 & 18 & 151 & 11.9 & 21 & 2 & 23 & 8.6 \\
\hline Total urogenital/rectal & 2172 & 26 & 2198 & 1.2 & 261 & 2 & 263 & 0.76 \\
\hline$\%$ Positive & & & 6.9 & & & & & 8.8 \\
\hline GC Negative & 2206 & 9 & 2215 & 0.41 & 246 & 3 & 249 & 1.2 \\
\hline GC Positive & 4 & 8 & 12 & 67 & 12 & 5 & 17 & 29 \\
\hline Total urogenital/rectal & 2210 & 17 & 2227 & 0.76 & 258 & 8 & 266 & 3.0 \\
\hline$\%$ Positive & & & 0.53 & & & & & 6.4 \\
\hline
\end{tabular}


Methods Retrospective case note review of $100 \mathrm{MSU}$ requests at a sexual health clinic between 2014 and 2015. The associated clinical presentations and culture results were identified.

Results 14\% of MSU were requested within guidelines. 29\% (4/ $14)$ of those were positive, compared to $22 \%$ (19/86) not requested within guidelines. Indications outside guidelines associated with positive culture included: women with lower urinary tract symptoms (11), men with dysuria only (3), pelvic inflammatory disease (2), asymptomatic with positive urine dipstick (2), and vaginal discharge (1). 15/23 were sensitive and 8/23 were resistant to trimethoprim.

Discussion/conclusion MSU is often requested inappropriately. This generates positive results associated with clinical presentations unlikely to indicate UTI. Greater awareness amongst clinicians of appropriate indications for MSU will support optimal resource utilisation in sexual health clinics. Resistance to our first line antibiotic, trimethoprim, was identified. Resistance patterns should be monitored so clinicians can confidently prescribe empirical treatment for lower UTI in nonpregnant women.

\section{P029 HIS-UK CONDOM STUDY: AN INTERVENTION DEVELOPMENT STUDY WORKING WITH YOUNG MEN TO IMPROVE CONDOM USE SKILLS AND ENHANCE CONDOM USE EXPERIENCES}

${ }^{1}$ Cynthia Graham, ${ }^{1}$ Sydney Anstee*, ${ }^{1}$ Nicole Stone, ${ }^{2}$ Katherine Brown, ${ }^{2}$ Katie Newby, ${ }^{1}$ Roger Ingham. ${ }^{1}$ University of Southampton, Southampton, UK; ${ }^{2}$ Coventry University, Coventry, UK

\subsection{6/sextrans-2016-052718.83}

Background The Kinsey Institute ${ }^{\circledR}$ Homework Intervention Strategy (KIHIS), designed to improve condom skills, enjoyment and self-efficacy, has demonstrated early evidence of efficacy in U.S. studies. The KIHIS places the impetus for change on the individual through solitary practice: experimenting with different condoms/lubricants; identifying best 'fit $\&$ feel'; and focusing on physical sensations.

Aim(s)/objectives To identify behaviour change techniques (BCTs) in KIHIS; to adapt and develop KIHIS for the UK context; to manualise and evaluate HIS-UK.

Methods Literature synthesis to identify additional BCT components and methods of delivery to address condom fit and feel. Stakeholder and user consultation through qualitative interviews ( $\mathrm{n}=15$ men aged 16-25); advisory groups (e.g. consultants, commissioners); workshops (e.g. health promotion professionals)

Results Searches of online databases, July 2015, identified 1044 condom use intervention studies published since 2006; of these, 123 studies tested the effectiveness of behavioural interventions on condom use in high income countries - and only five targeted 'fit \& feel' issues. In total 22 BCTs were identified, 16 of which were selected for inclusion in HIS-UK. Consultations have demonstrated enthusiasm for this 'fit \& feel' approach, have enabled us to gauge UK preferences (e.g. condom kit contents) and have informed adaptation of the intervention.

Discussion This work ensures that the targeted outcomes, behaviour determinants and proposed mechanisms of action for HISUK are specified, so that future conclusions can be drawn about what works and why. An adapted and manualised intervention is currently being piloted for viability and operability among 50 men aged $16-25$ years.

\section{P030 DEVELOPMENT, IMPLEMENTATION AND EARLY EVALUATION OF A PILOT CERVICAL SCREENING CLINIC FOR WOMEN WHO HAVE BEEN SEXUALLY ASSAULTED}

${ }^{1}$ Jill Zelin*, ${ }^{2}$ Louise Cadman, ${ }^{1}$ Jane Vosper, ${ }^{3,1}$ Pavan Amara. ${ }^{1}$ Barts Sexual Health Centre, St. bartholomew's Hospital, Barts Health, London, UK; ${ }^{2}$ Centre for Cancer Prevention, Wolfson Institute of Preventive Medicine, Queen Mary University of London, London, UK; ${ }^{3}$ My Body Back Project, London, UK

\subsection{6/sextrans-2016-052718.84}

Background One in five women does not attend for cervical screening when invited. This includes those who have experienced sexual violence, putting them at increased risk of cervical cancer. A pilot clinic was set up in partnership with the My Body Back Project (MBB). MBB supports women who have been raped to regain confidence and control of their body and health. The clinic offers cervical screening and STI testing for these women with a multidisciplinary collaboration between doctor, nurse, psychologist and $\mathrm{MBB}$ advocate as facilitator. It aims to provide time, space, shared control and understanding of the particular difficulties faced.

Aim To evaluate acceptability and uptake of a pilot cervical screening clinic for women with a history of sexual assault.

Methods Questionnaires were collected from women attending between August and December 2015.

Results 30 women attended (median age 34.4 years). $48.3 \%$ had never been screened and $72.4 \%$ were significantly overdue.

\begin{tabular}{|c|c|c|c|}
\hline Cytology result & $\begin{array}{l}\text { Cytology at visit } 1 \\
\left(\mathrm{~N}=26^{*}\right)\end{array}$ & $\begin{array}{l}\text { Cytology at visit } 2 \\
(\mathrm{~N}=3)\end{array}$ & $\begin{array}{l}\text { Total (\%) } \\
\text { smears taken }\end{array}$ \\
\hline Negative & 21 & 2 & $23(79.3)$ \\
\hline Unsatisfactory & 1 & 0 & 1 (3.4) \\
\hline Borderline HPV+ & 1 & 0 & $1(3.4)$ \\
\hline Results pending & 3 & $1^{* *}$ & $4.13 .8)$ \\
\hline $\begin{array}{l}\text { No cytology } \\
\text { taken }\end{array}$ & $2^{* * *}$ & 0 & $x$ \\
\hline TOTAL & 26 & 3 & $29(100)$ \\
\hline
\end{tabular}

Feedback showed $96.7 \%$ of women found the clinic very useful, the advocate helpful and felt understood. $86.2 \%$ found the smear taker and psychologist together helpful and 100\% would recommend the service. Confidence in their ability to have a smear increased from slightly/in some situations before their examination to in some/most situations afterwards. Common qualitative themes included not feeling rushed, feeling in control and having needs understood.

Discussion/conclusion The uptake, waiting list and feedback from women suggest that this is a necessary and appreciated clinic. Further evaluation is required in order to improve and sustain the service.

\section{P031 ESTIMATING LOCAL CHLAMYDIA INCIDENCE AND PREVALENCE USING SURVEILLANCE DATA}

1,2Joanna Lewis* ${ }^{1,2}$ Peter White. 'Department of Infectious Disease Epidemiology, Imperial College London, London, UK; ${ }^{2}$ Modelling and Economics Unit, Public Health England, London, UK

10.1136/sextrans-2016-052718.85 